\title{
Membrane Enhanced Peptide Synthesis
}

\author{
Sheung So, ${ }^{a}$ Ludmila G. Peeva, ${ }^{a}$ Edward W. Tate, ${ }^{b}$ Robin J. Leatherbarrow, ${ }^{b}$ Andrew G. Livingston ${ }^{a}$ \\ Received (in $X X X, X X X)$ Xth $X X X X X X X X X 200 X$, Accepted Xth $X X X X X X X X X 200 X$ \\ First published on the web Xth $X X X X X X X X X 200 X$ \\ ${ }_{5}$ DOI: 10.1039/b000000x
}

This paper reports a new technology platform that advantageously combines organic solvent nanofiltration (a newly emerging technology capable of molecular separations in organic solvents) with solution phase peptide synthesis - Membrane ${ }_{10}$ Enhanced Peptide Synthesis (MEPS).

In the last decade the market for peptide based pharmaceuticals has been growing rapidly: as of 2005 an estimated 40 peptide-based pharmaceutical products were for sale, with approximately 270 more in different phases of ${ }_{15}$ clinical trials ${ }^{1}$. Large-scale peptide manufacture is essential to bring these drugs to market. Solid-phase peptide synthesis (SPPS) is the most widely used technology, since it neatly solves the critical purification problems encountered at each stage in solution phase synthesis. However it faces serious 20 challenges including mass transfer, steric hindrance, and resin handling ${ }^{2,3,4}$. The concept of membrane separation coupled to solution phase synthesis offers major advantages over SPPS by combining the advantages of "classical" solution phase synthesis with the ease of purification of the solid phase 25 method ${ }^{2}$. Compared to SPPS, reactions in solution phase provide faster rates, and are less affected by steric hindrance due to peptide folding, or reactions within confined space which result in transpeptidation, and are not limited by intraparticle diffusional mass transfer phenomena ${ }^{3,4}$. This 30 enables reduction in the large reactant excesses, in some cases of up-to 5-fold ${ }^{5,6}$, that are employed in SPPS to compensate for mass transfer limitations. Solution phase reactions are also easier to scale-up due to the absence of swelling effects and cake formation within solid resins, which require more 35 complex reactor design. Prior applications of membrane separation in peptide chemistry are restricted to reconcentration of peptides ${ }^{7}$, amino acid recovery ${ }^{8,9}$, and a single report on membrane separation for purification between reaction cycles during peptide synthesis ${ }^{2,10}$. In the latter case, 40 peptides built on poly(ethylene glycol) (PEG) were separated from impurities by ultrafiltration. However, due to a lack of organic solvent-compatible membranes, this required evaporation of organic solvent after each coupling and each deprotection step, neutralisation after deprotection, and uptake 45 in water prior to ultrafiltration. Water was then removed by evaporation and/or azeotropic distillation before re-dissolving the PEG-peptide into organic solvent for the next coupling step. This complex process limited the synthesis to volatile organic solvents, and the lengthy solvent switching system 50 made the separation process unsuitable for large-scale production. Molecular separation in organic solvents via nanofiltration (Organic Solvent Nanofiltration - OSN) is an emerging technology ${ }^{11}$, which should be an ideal separation method for in-cycle purification during peptide synthesis.

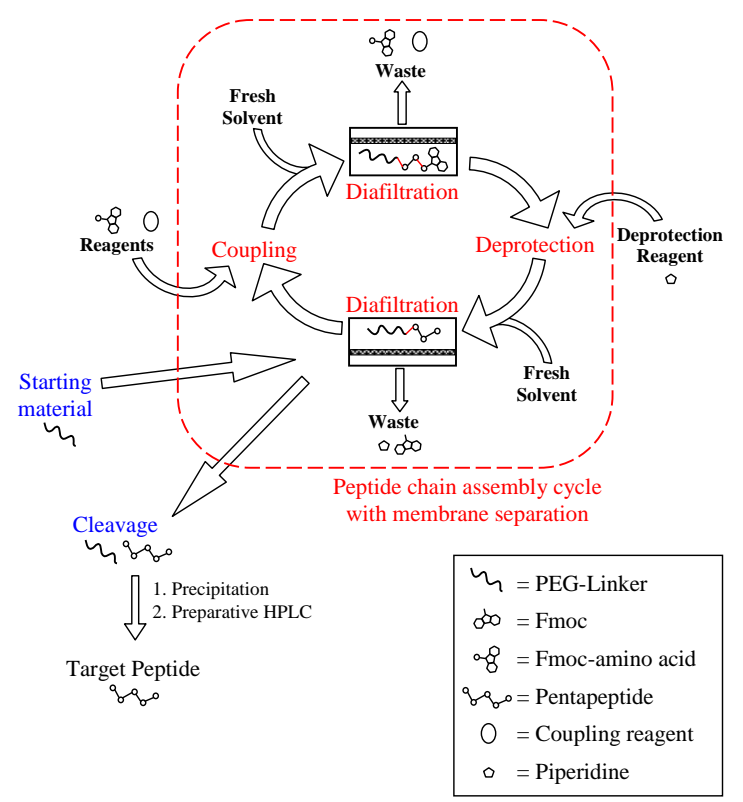

Figure 1: Schematic representation of membrane enhanced peptide synthesis (MEPS). Peptide chain assembly was performed following this scheme using the apparatus presented in Figure 4.

- Here we report for the first time Membrane Enhanced ${ }_{60}$ Peptide Synthesis (MEPS), a new technology platform that advantageously combines OSN with solution phase peptide synthesis.

The MEPS concept is illustrated in Figure 1. Peptide chain assembly occurs via: 1) amide coupling; 2) a washing step for 65 removal of excess reagents via constant volume diafiltration; 3) deprotection; 4) a washing step for removal of deprotection by-products and excess reagents again via diafiltration. The cycle is repeated as many times as necessary, adding a further amino acid each cycle, until the desired peptide sequence is 70 obtained. In contrast to the previously reported studies 2,10 washing is carried out immediately after the coupling and deprotection steps using the reaction solvent, and does not require any solvent exchanges.

The peptide is assembled on a soluble polymeric support, 75 methoxy-amino-PEG with molecular weight (MW) 5,000 g. $\mathrm{mol}^{-1}\left(\mathrm{MeO}-\mathrm{PEG}-\mathrm{NH}_{2}\right)$, to increase retention by the membrane ${ }^{3}$. Since PEGylated peptides have received much recent attention due to their enhanced therapeutic and pharmacokinetic potential ${ }^{12-14}$, in some cases a bioactive 80 peptide made by MEPS might also be used directly without cleavage from PEG. 
Fmoc (9-fluorenylmethoxycarbonyl) peptide synthesis chemistry was chosen due to its widespread application and mild deprotection conditions (piperidine/DMF). Hydroxymethylphenylacetic acid (HMPA) was used as linker 5 to attach the first amino acid in the peptide sequence to the MeO-PEG- $\mathrm{NH}_{2}$ enabling facile cleavage of the final peptide via acidolysis. Peptide coupling proceeds optimally in polar aprotic solvents such as dimethylformamide (DMF) or $\mathrm{N}$ methyl pyrrolidinone (NMP), and DMF was used in both 10 reaction and diafiltration steps. The use of these solvents in combination with OSN membranes has only recently become possible with the development of membranes ${ }^{15}$ which possess good stability in these aprotic solvents and make this approach viable.

15 The most important consideration for successful realisation of this approach was the choice of membrane. This must possess excellent long term stability in the reaction solvent (DMF) and high selectivity between MeO-PEG-peptide, and side reaction products and excess reagents, including 20 unreacted amino acids, activators and deprotection reagents. Membrane performance should not be affected by frequent switching of the reaction media between DMF solution in the coupling step and $20 \%$ piperidine/DMF solution in the deprotection step.

25 After screening a range of commercial and developmental membranes we identified a ceramic OSN membrane that met these requirements: the Inopor $\mathrm{ZrO}_{2}$ coated membrane with $3 \mathrm{~nm}$ pore size and hydrophobic surface modification (Innocermic, Germany). Based on the membrane 30 characterisation data it was estimated that 10-12 solvent wash volumes should be sufficient for removal of all excess reagents from around 1.0 to less than 0.01 equivalents.

To prove the MEPS concept we started by producing a model peptide H-Tyr-Ala-Tyr-Ala-Tyr-OH. This was chosen 35 as it includes one of the largest protected amino acids Fmoc$\operatorname{Tyr}\left({ }^{t} \mathrm{Bu}\right)-\mathrm{OH}$, and one of the smallest hydrophobic amino acids, Fmoc-Ala-OH, thus providing information on the performance of the MEPS process with respect to different molecular sizes and properties of amino acids. PyBOP was 40 chosen for the coupling reaction as it is one of the largest of the commercial activators available, and its successful removal presents a challenge for the MEPS process. DIC was used for the esterification linking the first amino acid onto MeO-PEG-HMPA. Thus the synthesis also provided insight 45 into the behaviour of the post-reaction species derived from both activators during diafiltration. Finally, this first experiment also sought to establish membrane stability at high concentrations of organic base (piperidine) during the deprotection step (see Table S1, Supporting Information).

50 The model peptide produced contained no peptide byproducts as confirmed by RP-HPLC and MALDI-TOF analysis. This absence of any detectable peptide impurities indicates that membrane purification is efficient at removing un-reacted protected amino acids throughout the synthesis. ${ }_{55}$ Our calculations suggested that traces of impurities would still remain in the system after each purification step even after 10 volumes of washing solvent, but this did not affect the final peptide purity. Apparently the level of impurities that can be

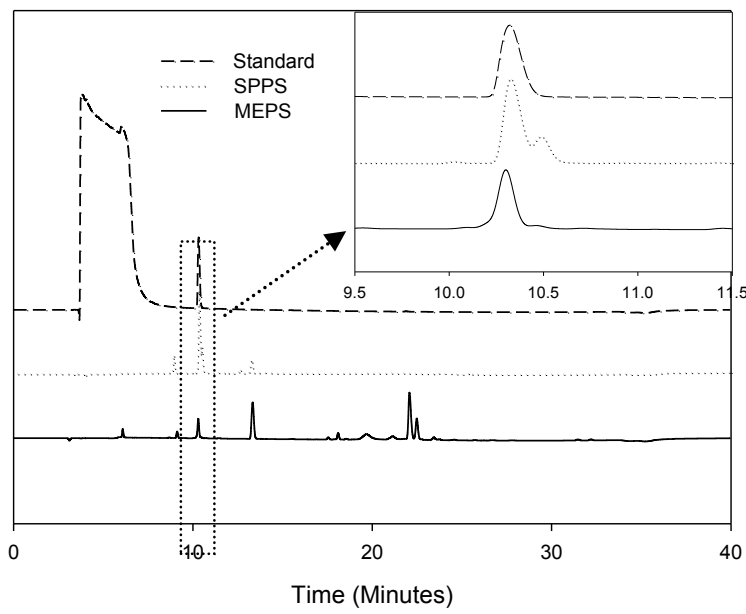

60 Figure 2: HPLC chromatograms of peptide TP-5 produced by MEPS and SPPS processes, and TP-5 standard purchased from Sigma-Aldrich (UK). The target TP-5 peptide was eluted at 10.3 minutes. Both syntheses (MEPS and SPPS) were performed under the same reaction conditions of 2 equivalents of reagents per 1 equivalent peptide, and single reaction 65 cycles. Peptide purity was determined as a ratio between the target peptide TP-5 peak area and the total area of the peaks corresponding to peptide sequences in the solution. The purity of TP-5 produced by MEPS was determined as $\sim 94 \%$ (two impurities eluted at 10.0 minutes and 10.4 minutes) while TP-5 produced by SPPS was $\sim 77 \%$ pure (one impurity 70 eluted at 10.5 minutes). The large peaks eluted between $19-23$ minutes were PEGylated wastes such as MeO-PEG-HMPA and the peak eluted at 13 minutes was not of peptide origin as confirmed by MALDI-TOF analysis and were not taken into account for the purity calculations.

tolerated in the system without provoking side reactions is 75 higher than anticipated, and so there is potential to reduce the washing volume.

Encouraged by this first success, Thymopentin (H-Arg-LysAsp-Val-Tyr-OH) was synthesized as a second demonstration of the MEPS process. Thymopentin (TP-5) is a derivative of 80 the naturally occurring hormone thymopoietin, with potential for the treatment of rheumatoid arthritis, AIDS and other primary immunodeficiencies ${ }^{16}$. Besides being a potential active pharmaceutical, this peptide includes a range of amino acids from aromatic (Tyr), acidic (Asp) and basic (Lys and ${ }_{85} \mathrm{Arg}$ ) to hydrophobic (Val). It also contains the largest Fmoc/Boc protected amino acid (Fmoc-Arg(Boc) $)_{2}$, M.W. 597 g. $\mathrm{mol}^{-1}$, and so this synthesis represents a significant further challenge for MEPS. RP-HPLC analyses of TP-5 produced by both MEPS and SPPS are illustrated in Figure 2.

90 The purity of the MEPS product (as a percentage of total (TP-5 plus peptidic by-products)) was estimated at $94 \%$. MALDI-TOF analysis (Figure 3) confirmed the target product molecular weight of $\mathrm{MH}+680$ and identified the two impurities as formed by deletion of Asp, MH+ 564, and Lys, ${ }_{95} \mathrm{MH}+550$. TP-5 produced by SPPS under the same reagent excess (2 equivalents) was only $77 \%$ pure and the main impurity was identified as deletion of Arg, MH+ 524. This result demonstrates the key advantage of liquid phase synthesis over SPPS - a higher purity was obtained using the 100 same excess of reagents. Typically more equivalents are needed for SPPS ${ }^{5}$. The overall yield of TP-5 produced by the MEPS process was estimated to be $92 \%$, with respect to the starting MeO-PEG- $\mathrm{NH}_{2}$ material. 


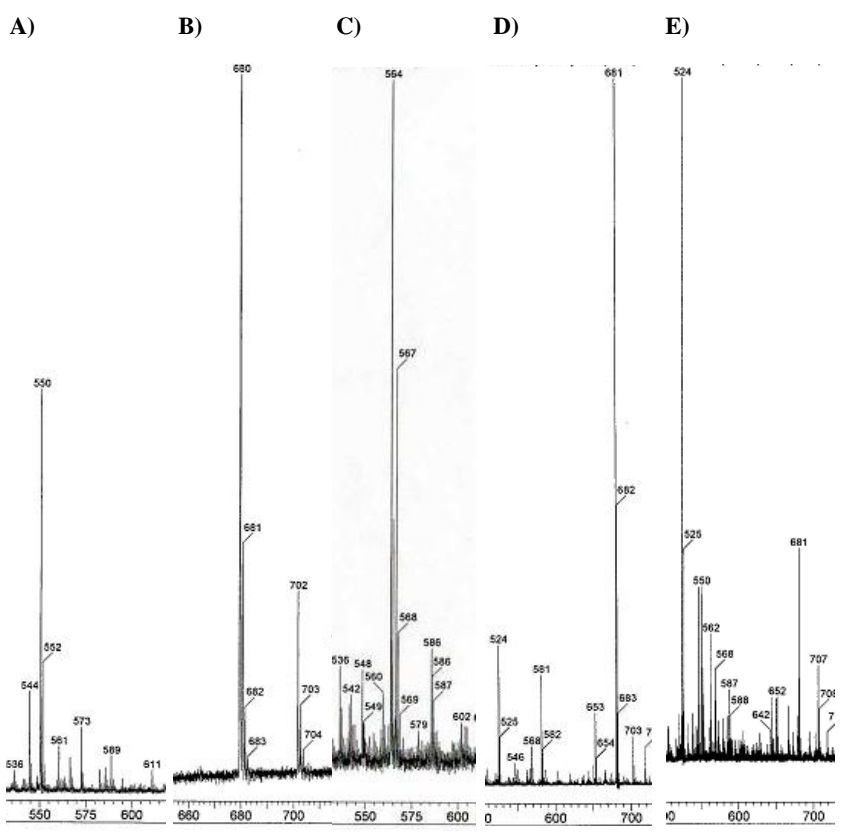

Figure 3: MALDI-TOF mass spectrum of the different peptides produced by MEPS and SPPS TP-5 synthesis and isolated using semi-preparative 5 HPLC. Spectrum B) - from the MEPS process and D) - from the SPPS process correspond to the peak eluted at 10.3 minutes. It was identified as TP-5 and showed the target molecular mass, MH+ of $680 \mathrm{Da}$. Spectrum A) and C) - from the MEPS process correspond to the two impurities eluted at 10.0 and 10.4 minutes respectively and were identified as 10 deletion of Lys, MH+ $550 \mathrm{Da}$ and Asp, MH+ $564 \mathrm{Da}$. Spectrum E) from the SPPS process corresponds to the impurity eluted at 10.5 minutes and was identified as deletion of $\mathrm{Arg}, \mathrm{MH}+524 \mathrm{Da}$.
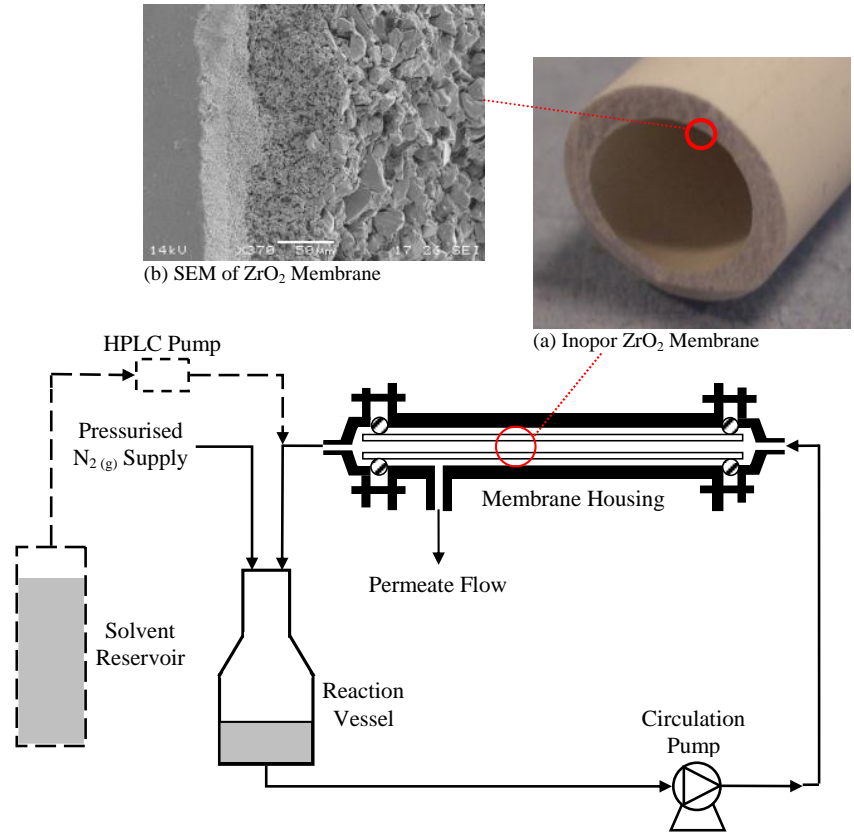

Figure 4: Experimental set-up used for peptide chain assembly: Both 15 coupling and deprotection reactions were performed in the Reaction Vessel where mixing was provided via the Circulation Pump. Upon completion of each reaction, the system was pressurised to 7 barg using nitrogen gas. Fresh DMF solvent was pumped via an HPLC pump from the Solvent Reservoir into the system to replace the permeated solvent 20 and maintain constant liquid volume within the Reaction Vessel; (a) Inopor $\mathrm{ZrO}_{2}$ ceramic membrane - front view; (b) SEM image of the Inopor $\mathrm{ZrO}_{2}$ ceramic membrane - edge view at magnification 370x. The line bar corresponds to $50 \mu \mathrm{m}$.
For this investigation batch size of $0.9 \mathrm{mmol}$ TP-5 was produced which yielded $\sim 0.6 \mathrm{~g}$ of product respectively. With the current laboratory set-up (Figure 4) $>20 \mathrm{mmol}$ batches of 25 peptide can be produced by simply increasing the feed volume and using identical operating conditions. Further scale-up to kilogram or ton scale may be possible by increasing the size of the equipment.

The MEPS process proposed in this work integrates the 30 advantages of performing peptide synthesis in solution with a direct membrane purification of the post-reaction mixture. The process is less constrained by mass-transfer limitations, and requires a smaller excess of reagents, than SPPS, yet demonstrates excellent purity and yield of the final peptide. ${ }_{35} \mathrm{We}$ anticipate that further optimisation of the separation step and wash solvent volume will result in solvent savings and improved process economics. Thus, we conjecture that the MEPS process offers an important alternative technology platform for peptide and PEGylated peptide production at 40 industrial scale.

This work was funded by the U.K. Engineering and Physical Sciences Research Council (EPSRC) Grant NoEP/C50982X/1; The authors would like to acknowledge also the financial support from the FP7 IAPP EC Grant ${ }_{45}$ NoPIAP-GA-2008-218068.

\section{Notes and references}

${ }^{a}$ Department of Chemical Engineering, Imperial College London, London SW7 2AZ, UK. Fax: +44 (0)20 75945629; Tel: +44 (0)20 75945582; E-mail: a.livingston@imperial.ac.uk

${ }_{50}{ }^{b}$ Department of Chemistry, Imperial College London, London SW7 2AZ,

UK.E-mail e.tate@imperial.ac.uk

$\dagger$ Electronic Supplementary Information (ESI) available: Experimental Methods and Procedures; Peptide Synthesis; Analysis ; Peptide purity and yield. See DOI:

1 B. Bray, Nature Review Drug Discovery, 2003, 2, 587 - 593.

2 E. Bayer, M. Mutter, Nature, 1972, 237, $512-513$.

3 E. Bayer, M. Mutter, R. Uhmann, J. Polster, H. Mauser, J. Am. Chem. Soc., 1974, 96, 7333-7336.

604 V. Gut, J. Rudinger in Peptides 1968, (Eds.: E. Bricas) Amsterdam, 1968, 185-188.

5 M. Amblard, J. Fehrentz, J. Martinez, G. Subra, Mol. Biotech., 2006, 33, $239-254$.

6 W. Chan, P. White, Fmoc solid phase peptide synthesis: a practical approach, Oxford University Press, 2000.

7 T. Tsuru, S. Nakao, S. Kimura, T. Shutou, Sep. Sci. Technol., 1994, 29, $971-984$

8 S-L. Li, C. Li , Y-S. Lui, X-L. Wang, Z-A. Cao, J. Membr. Sci., 2003, 222, $191-201$

709 X. Wang, A. Ying, W. Wang, J. Membr. Sci., 2002, 196, 59 - 67.

10 E. Bayer, D. Gillessen, H. Kuenzi, M. Mutter, R. Studer (F. Hoffmann-La Roche \& Co.), DE-F2047413, 1980.

11 P. Vandezande, L.E.M. Gevers, I.F.J. Vankelecom, Chem. Soc. Rev., 2008, 37, 365-405.

7512 M. Hamidi, A. Azadi, P. Rafiei, Drug Delivery., 2006, 13, 399 - 409.

13 S.-H. Lee, S. Lee, Y. Youn, D. Na, S. Chae, Y. Byun, K. Lee, Bioconjugate Chem., 2005, 16, $377-382$.

14 M. Roberts, M. Bentley, J. Harris, Adv. Drug Delivery Rev., 2002, 54, $459-476$

8015 Y. See Toh, F. Lim, A. Livingston, J. Membr. Sci., 2007, 301, 3-10.

16 E. Ochoa, S. Medrano, M. de Carlin, A. Dilonardo, Cell. Mol. Neurobiol., 1988, 8, 325 - 331 . 